\title{
Characterization of lymphoblast mitochondria from patients with Barth syndrome
}

\author{
Yang $\mathrm{Xu}^{1}$, John J Sutachan ${ }^{1}$, Heide Plesken ${ }^{2}$, Richard I Kelley ${ }^{3}$ and Michael Schlame ${ }^{1}$ \\ ${ }^{1}$ Department of Anesthesiology, New York University School of Medicine, New York, NY, USA; ${ }^{2}$ Department \\ of Cell Biology, New York University School of Medicine, New York, NY, USA and ${ }^{3}$ Division of Metabolism, \\ Kennedy Krieger Institute, Baltimore, MD, USA
}

\begin{abstract}
Barth syndrome (BTHS) is a multisystem disorder of individuals who carry mutations in tafazzin, a putative phospholipid acyltransferase. We investigated the hypothesis that BTHS is caused by specific impairment of the mitochondrial lipid metabolism. The fatty acid composition of all major mitochondrial phospholipids, phosphatidylcholine (PC), phosphatidylethanolamine (PE), and cardiolipin (CL), changed in lymphoblasts from BTHS patients. These changes were most extensive in $\mathrm{CL}$ and least extensive in PE. The complementary nature of the fatty acid alterations in CL and PC suggested that fatty acid transfer between these two lipids was inhibited in BTHS. Fluorescence staining and electron microscopy showed abnormal proliferation of mitochondria in BTHS lymphoblasts. The mitochondrial membrane potential, monitored with the fluorescence probe JC-1, was reduced in BTHS lymphoblasts. However, mitochondrial ATP formation of permeabilized lymphoblasts remained unaffected in BTHS. The data suggest that phospholipid abnormalities of BTHS mitochondria led to partial uncoupling of oxidative phosphorylation and that lymphoblasts compensated for this deficiency by expanding the mitochondrial compartment.
\end{abstract}

Laboratory Investigation (2005) 85, 823-830, advance online publication, 4 April 2005; doi:10.1038/labinvest.3700274

Keywords: cardiomyopathy; fatty acid; mitochondria; oxidative phosphorylation; phospholipid

Barth syndrome (BTHS) is an X-linked disease presenting with cardiomyopathy, skeletal myopathy, neutropenia, and growth retardation. ${ }^{1-4}$ Mutations that cause BTHS are located in the tafazzin gene, ${ }^{5}$ which belongs to a large superfamily of phospholipid acyltransferases. ${ }^{6}$ The lipid etiology of BTHS was confirmed when Vreken et $a l^{7}$ found aberrant cardiolipin (CL) metabolism in BTHS fibroblasts. CL is a dimeric phospholipid that is specific to mitochondria and has a unique fatty acid pattern. ${ }^{8}$ The fatty acid pattern of CL is severely disturbed in heart, skeletal muscle,${ }^{9,10}$ platelets, ${ }^{9-11}$ and neutrophils of BTHS patients. ${ }^{12}$ The same phenomenon was observed in tafazzin-deficient yeast. ${ }^{13,14}$ It appears that tafazzin is required to generate mature CL by remodeling its fatty acid pattern. ${ }^{15}$ In the absence of functional tafazzin, CL is unable to form certain molecular species (specific combinations of fatty acids in a single molecule), the concentration

Correspondence: Dr M Schlame, MD, Department of Anesthesiology, New York University School of Medicine, 550 First Avenue, New York, NY 10016, USA.

E-mail: michael.schlame@med.nyu.edu

Received 13 January 2005; revised 12 February 2005; accepted 15 February 2005; published online 4 April 2005 of CL is reduced, and there is accumulation of its degradation product, monolyso-CL. ${ }^{9-15}$

BTHS is unique because it is the only cardiomyopathy whose origin is found in phospholipid metabolism. However, the molecular mechanism of the disease has not been understood. In particular, it has remained elusive how the phospholipid defect relates to any of the established pathways to cardiomyopathy, which involve either the contractile apparatus or the energy supply. ${ }^{16,17}$ The CL changes suggest that mitochondria may be the primary site of the lipid defect and that BTHS may affect mitochondrial function. In this paper, we tested this hypothesis in lymphoblasts from children with BTHS. We studied phospholipid patterns inside and outside of mitochondria and characterized mitochondrial properties related to oxidative phosphorylation.

\section{Materials and methods}

\section{Patients}

The study was performed with lymphoblasts from five patients with BTHS and five control subjects (age 1-9 years). All patients were male. BTHS patients had mutations in the tafazzin gene, which 
caused protein deletion. They presented with cardiomyopathy plus at least two of the noncardiac symptoms of BTHS (neutropenia, growth retardation, skeletal muscle weakness, 3-methylglutaconic aciduria). Control patients were individuals with normal tafazzin genes who presented for work-up of unrelated neurologic conditions. The human study protocol was approved by institutional review boards of Johns Hopkins University and New York University Medical Center.

\section{Lymphoblast Cultures and Isolation of Subcellular Membranes}

Lymphoblast cell lines were established by EpsteinBarr virus transformation of leukocytes isolated from whole blood using Ficoll-Hypaque gradients. The cell lines were cultured in RPMI 1640 medium in the presence of fetal bovine serum $(10 \%)$, penicillin $(50 \mathrm{IU} / \mathrm{ml})$, and streptomycin $(50 \mu \mathrm{g} / \mathrm{ml})$ at $37^{\circ} \mathrm{C}$ under $5 \% \mathrm{CO}_{2}$ atmosphere. The serum was heat-inactivated at $56^{\circ} \mathrm{C}$ for $30 \mathrm{~min}$ prior to use. Lymphoblasts were seeded at a density of $3 \times 10^{5}$ cells/ml and cultures were expanded every 2-3 days. Mitochondria were isolated from lymphoblasts, growing at mid-log phase, at $4^{\circ} \mathrm{C}$ according to a published protocol. ${ }^{18}$ In short, $1-2 \times 10^{9}$ cells, growing in mid-log phase, were harvested and their wet weight was determined. Cells were resuspended in chilled isolation buffer $(210 \mathrm{mM}$ mannitol, $70 \mathrm{mM}$ sucrose, $1 \mathrm{mM}$ EDTA, $0.5 \%$ fatty acid-free bovine serum albumin, $5 \mathrm{mM}$ HEPES, $\mathrm{pH}$ 7.2) at a concentration of $4 \mathrm{ml}$ per gram wet weight. Digitonin $(10 \%$ $\mathrm{w} / \mathrm{v}$ in DMSO) was slowly added to a final concentration of $0.2-0.4 \mathrm{mg} / \mathrm{ml}$ until more than $90 \%$ of lymphoblasts were permeabilized. Permeabilization was determined by staining with trypan blue. The digitonin incubation was terminated by dilution with isolation buffer. Permeabilized cells were collected by centrifugation and disrupted in a tight-fitting glass-Teflon homogenizer. Cell debris and nuclei were removed by three runs of 5 -min spins at $625 \mathrm{~g}$. The final supernatant was spun at $10000 \mathrm{~g}$ for $20 \mathrm{~min}$, yielding the mitochondrial pellet. The post-mitochondrial supernatant was spun at $100000 \mathrm{~g}$ for $1 \mathrm{~h}$, yielding the microsomal pellet. The membranes were re-suspended in buffer and stored at $-80^{\circ} \mathrm{C}$.

\section{Fatty Acid Composition of Phospholipids}

Lipids were extracted from whole lymphoblasts, lymphoblast mitochondria, or microsomes according to Bligh and Dyer. ${ }^{19}$ Extracted lipids were separated by two-dimensional thin-layer chromatography on silica gel 60, developed with chloroformmethanol-water $(65 / 25 / 4)$ in the first direction and chloroform-acetone-methanol-acetic acid-water $(50 / 20 / 10 / 10 / 5)$ in the second direction. Lipids were stained by brief exposure to iodine. The spots of phosphatidylcholine (PC), phosphatidylethanolamine (PE), and CL were marked and iodine was blown off. The spots were scraped into reaction vials for transmethylation, which was performed in the presence of $0.5 \mathrm{M} \mathrm{HCl} /$ methanol at $90^{\circ} \mathrm{C}$ for $12 \mathrm{~h}$. The reaction mixture was neutralized with saturated $\mathrm{NaHCO}_{3}$ and extracted with $n$-hexane. Fatty acid methyl esters were analyzed by isothermic $\left(185^{\circ} \mathrm{C}\right)$ gas chromatography, using a Shimadzu GC-17A instrument equipped with a SP-2330 capillary column $(0.32 \mathrm{~mm} \times 30 \mathrm{~m}, 0.2 \mu \mathrm{m}$ column film) and a flame ionization detector.

\section{Molecular Species of PC}

PC was isolated from lymphoblasts by two-dimensional thin-layer chromatography as described above. It was extracted from silica gel with chloroform-methanol (1/1) and treated with phospholipase C from Bacillus cereus. To this end, PC was dissolved in $1 \mathrm{ml}$ diethylether-ethanol (95/5) and stirred for $8 \mathrm{~h}$ in the presence of $0.5 \mathrm{ml}$ aqueous buffer containg $0.03 \mathrm{M} \mathrm{H}_{3} \mathrm{BO}_{3}, 0.25 \mathrm{M}$ HEPES ( $\mathrm{pH}$ 7.2 ), and $15 \mathrm{U}$ of enzyme. Diacylglycerol was collected in the ether phase and derivatized with 3,5-dinitrobenzoylchloride in dry pyridine at $70^{\circ} \mathrm{C}$ for $15 \mathrm{~min} .{ }^{20}$ Molecular species of 1,2-diacyl-3-( $3^{\prime}, 5^{\prime}-$ dinitrobenzoyl)glycerol were resolved by reversedphase high-performance liquid chromatography on a Nucleosil C18 column $(4.6 \times 250 \mathrm{~mm}, 5 \mu \mathrm{m}$ particles) eluted with acetonitrile-2-propanol (4/1). The flow rate was $1 \mathrm{ml} / \mathrm{min}$. Chromatograms were recorded by a multiple wavelength UV/VIS detector set at $254 \mathrm{~nm}$. Molecular species were identified by comparison with standard compounds and by fatty acid analysis.

\section{Electron Microscopy}

Lymphoblast pellets were fixed with $2 \%$ glutaraldehyde in $0.1 \mathrm{M}$ sodium cacodylate buffer, $\mathrm{pH} 7.3$, for $3 \mathrm{~h}$. Pellets were post-fixed in $2 \%$ osmium tetroxide/ cacodylate buffer for $2 \mathrm{~h}$. Cells were in-block stained with $1.3 \%$ aqueous uranyl acetate for $30 \mathrm{~min}$. After dehydration in ethanol and substitution with propylene oxide, the samples were embedded in epoxy resin. Thin sections $(10 \mathrm{~nm})$ were collected on carbon-coated grids. They were contrasted with uranyl acetate and lead citrate. Images were taken with a JEOL JEM1200EXII electron microscope.

\section{Flow Cytometry and Imaging of Lymphoblasts}

One million lymphoblasts were incubated in $1.5 \mathrm{ml}$ culture medium with $0.5 \mu \mathrm{M}$ MitoTracker Red CMXRos (Molecular Probes, Eugene, OR, USA) at $37^{\circ} \mathrm{C}$, in $5 \% \mathrm{CO}_{2}$ atmosphere for $30 \mathrm{~min}$. The stained cells were washed twice with pre-warmed medium and fixed with $3.7 \%$ paraformaldehyde at $37^{\circ} \mathrm{C}$ for 
15 min. Thereafter cells were washed three times with phosphate-buffered saline. Single red color flow cytometry analysis was performed with a Beckton Dickinson FACS Calibur instrument. For image analysis, stained lymphoblasts were spread on glass slides mounted with the AQUA-poly/ mount medium (Polysciences, Inc., Warrington, PA, USA). Cells were illuminated with a $100 \mathrm{~W}$ mercury arc lamp (AttoArc ${ }^{\circledR} 2$ HBO $100 \mathrm{~W}$, ZEISS). Red fluorescence emissions were collected through a 63X Plan-APOCHROMAT, 1.40NA oil objective (ZEISS) using a standard HQ:TRITC/DIL filter set (CHROMA, Rockingham, VT, USA). The cells were imaged with a non-laser Atto Confocal Image System (ATTO Bioscience, Rockville, MD, USA), including an Axiovert 135 inverted fluorescence microscope (ZEISS, Oberkochen, Germany) equipped with a cooled CCD imaging digital camera with extended IR range and extra sensitivity (ORCAER Digital Camera, C4742-95, HAMAMATSU). Fluorescence emissions were captured as 12-bit images using the video sensor of the CCD digital camera at $1024 \times 1024$ pixel resolution and analyzed using AttoView software. Consecutive frames of about $0.2 \mu \mathrm{m}$ optical sections (Z-series) were collected with CARVer ${ }^{\circledR}$ and their confocal 3D images were reconstructed using LSM PC software (ZEISS).

\section{Mitochondrial Membrane Potential}

One million lymphoblasts, growing at mid-logarithmic phase, were incubated in $1.5 \mathrm{ml}$ culture medium with $7.5 \mu \mathrm{M}$ JC-1 $\left(5,5^{\prime}, 6,6^{\prime}\right.$-tetrachloro-1, 1' ${ }^{\prime}, 3,3^{\prime}$-tetraethylbenzimidazolyl-carbocyanine iodide, Molecular Probes, Eugene, OR, USA) for $10 \mathrm{~min}$ at $37^{\circ} \mathrm{C}$ in $5 \% \mathrm{CO}_{2}$ atmosphere. The JC-1 stained cells were washed three times with PBS, suspended in $1 \mathrm{ml}$ phosphate-buffered saline containing 3\% fetal bovine serum, and immediately analyzed by flow cytometry. The green fluorescence emission (JC-1 monomers) was monitored at $520 \mathrm{~nm}$ and the red-orange fluorescence emission (J-aggregates) was monitored at $590 \mathrm{~nm}$. The aggregate/monomer ratio was proportional to the mitochondrial membrane potential. To determine the reference signal of uncoupled mitochondria, cells were pretreated with $1 \mu \mathrm{M}$ carbonyl cyanide p-(trifluoromethoxy)phenylhydrazone for $5 \mathrm{~min}$ before staining.

\section{Mitochondrial ATP Formation}

Mitochondrial ATP synthesis was measured in permeabilized cells similar to a previously published protocol. ${ }^{21}$ Lymphoblasts $\left(10^{7}\right.$ cells $)$ were suspended in $1 \mathrm{ml}$ phosphate-buffered saline and permeabilized by incubation with $15 \mu \mathrm{g}$ digitonin for $1 \mathrm{~min}$ at room temperature. The reaction was stopped by dilution with phosphate-buffered saline. Permeabilized cells were collected by centrifugation and resuspended in $0.5 \mathrm{ml}$ oxphos medium, contain- ing $110 \mathrm{mM} \mathrm{KCl,} 5 \mathrm{mM}$ glutamic acid, $5 \mathrm{mM}$ succinic

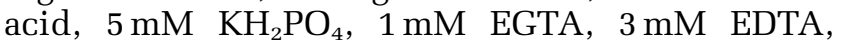
$10 \mathrm{mM}$ Tris, $25 \mu \mathrm{M} \mathrm{P}^{1} \mathrm{P}^{5}$-di(adenosine-5'-)pentaphosphate, and $2 \mathrm{mM}$ iodoacetate ( $\mathrm{pH} 7.4$ by $\mathrm{KOH}$ ). The suspension was divided into $60-\mu \mathrm{l}$ aliquots and ATP formation was initiated by addition of $100 \mathrm{nmol}$ ADP. Every measurement was accompanied by a control sample that contained $1 \mu \mathrm{g}$ oligomycin to inhibit oxidative phosphorylation. The incubation was stopped after $10 \mathrm{~min}$ by addition of $100 \mu \mathrm{l}$ $\mathrm{HClO}_{4}(14 \%)$. Precipitated protein was removed by centrifugation, $140 \mu \mathrm{l}$ of the supernatant were withdrawn and neutralized with $80 \mu \mathrm{l}$ of $1.6 \mathrm{M} \mathrm{Na}_{2} \mathrm{CO}_{3}$. The concentration of ATP was measured in the neutralized perchloric acid extracts by coupled enzyme assay using a fluorescence spectrophotometer. $^{22}$ ATP formation was shown to be linear with respect to incubation time. Mitochondrial ATP formation was calculated from the difference of ATP in the presence and absence of oligomycin.

\section{Statistical Analysis}

For each experiment, lymphoblast cultures from five individuals were measured in the control group and the BTHS group, respectively. Data were expressed as means with standard error of mean $(n=5)$. Comparisons between groups were performed by Student's $t$-test.

\section{Results}

\section{Analysis of Phospholipids}

Previously, we found reduced concentration and abnormal fatty acid composition of CL in various tissues from BTHS patients. ${ }^{10}$ Although the concentration of other phospholipids remained normal in BTHS, ${ }^{10}$ it was not known whether or not the fatty acid composition of these phospholipids was affected as well. In the present study, we provided a comprehensive fatty acid analysis of PC, PE, and CL in lymphoblasts from BTHS patients and controls (Table 1).

As expected, BTHS induced significant changes in the CL acyl pattern. In particular, palmitoleic (16:1) and linoleic (18:2) acid were replaced by palmitic (16:0) and stearic (18:0) acid. Furthermore, there was a shift from oleic acid $\left(18: 1 \Delta^{9}\right)$ to vaccenic acid $\left(18: 1 \Delta^{11}\right)$, as a result of which vaccenic acid became the dominant acyl group in CL from BTHS lymphoblasts.

We also found some alterations in the fatty acid composition of PC, most notably an increase in palmitoleic acid at expense of oleic acid (Table 1). Analysis of the PC molecular species showed an increase of 16:0-16:1-PC at the expense of 16:018:1-PC in BTHS lymphoblasts (Figure 1). The BTHS-associated changes of PC were present in both the mitochondrial and the microsomal fraction. 
Table 1 Fatty acid composition of lymphoblast phospholipids

\begin{tabular}{|c|c|c|c|c|c|c|}
\hline \multirow[t]{2}{*}{ Fatty acid } & \multicolumn{2}{|c|}{$P C$} & \multicolumn{2}{|c|}{$P E$} & \multicolumn{2}{|c|}{$C L$} \\
\hline & Control & BTHS & Control & BTHS & Control & BTHS \\
\hline $14: 0$ & $2.2 \pm 1.3$ & $3.5 \pm 0.2$ & $3.2 \pm 0.3$ & $3.5 \pm 0.4$ & $1.8 \pm 0.3$ & $2.5 \pm 0.2$ \\
\hline $16: 0$ & $25.8 \pm 2.6$ & $30.9 \pm 2.3$ & $4.3 \pm 2.0$ & $8.4 \pm 1.3$ & $6.1 \pm 0.7$ & $19.5 \pm 0.4^{\mathrm{a}}$ \\
\hline $16: 1$ & $6.9 \pm 1.2$ & $12.4 \pm 0.9^{\mathrm{a}}$ & $2.9 \pm 0.1$ & $4.9 \pm 0.9^{\mathrm{a}}$ & $15.5 \pm 0.9$ & $5.4 \pm 1.0^{\mathrm{a}}$ \\
\hline 18:0 & $11.5 \pm 2.1$ & $9.2 \pm 2.9$ & $23.2 \pm 1.5$ & $23.2 \pm 1.4$ & $1.5 \pm 0.1$ & $5.7 \pm 0.1^{\mathrm{a}}$ \\
\hline $18: 1 \Delta^{9}$ & $33.1 \pm 3.9$ & $24.2 \pm 2.8^{\mathrm{a}}$ & $26.4 \pm 2.3$ & $25.3 \pm 2.8$ & $21.8 \pm 4.3$ & $10.0 \pm 1.1^{\mathrm{a}}$ \\
\hline $18: 1 \Delta^{11}$ & $7.5 \pm 1.6$ & $8.0 \pm 0.4$ & ND & ND & $37.0 \pm 3.0$ & $55.5 \pm 1.1^{\mathrm{a}}$ \\
\hline $18: 2$ & $2.0 \pm 0.8$ & $1.6 \pm 0.2$ & $1.3 \pm 0.2$ & $1.9 \pm 0.1$ & $12.3 \pm 1.0$ & $1.4 \pm 0.4^{\mathrm{a}}$ \\
\hline $20: 1$ & $3.2 \pm 0.2$ & $1.5 \pm 0.1^{\mathrm{a}}$ & $1.7 \pm 0.1$ & $1.0 \pm 0.1$ & $\mathrm{ND}$ & ND \\
\hline $20: 2$ & $1.6 \pm 0.2$ & $2.0 \pm 0.1$ & $1.1 \pm 0.1$ & $1.6 \pm 0.1$ & ND & ND \\
\hline $20: 3$ & $2.0 \pm 0.4$ & $2.2 \pm 0.8$ & $6.4 \pm 2.1$ & $6.5 \pm 0.1$ & $3.9 \pm 0.4$ & $0.1 \pm 0.1^{\mathrm{a}}$ \\
\hline $20: 4$ & $2.1 \pm 0.4$ & $2.3 \pm 1.2$ & $12.9 \pm 0.2$ & $11.9 \pm 3.0$ & $\mathrm{ND}$ & $\overline{N D}$ \\
\hline $22: 5$ & $0.6 \pm 0.1$ & $0.6 \pm 0.2$ & $4.4 \pm 0.8$ & $2.8 \pm 0.6$ & ND & ND \\
\hline $22: 6$ & $0.7 \pm 0.1$ & $0.8 \pm 0.3$ & $7.0 \pm 0.6$ & $4.9 \pm 1.5$ & ND & ND \\
\hline Others & $0.8 \pm 0.2$ & $0.7 \pm 0.3$ & $5.5 \pm 0.5$ & $3.9 \pm 1.3$ & ND & ND \\
\hline
\end{tabular}

Fatty acid composition is given in mass\%. Fatty acids are abbreviated as $x: y$, where $x$ represents the number of carbon atoms and $y$ represents the number of double bonds.

${ }^{\mathrm{a}}$ Significant difference between control and BTHS $(P<0.05)$.

18:1 $\Delta^{9}$, oleic acid; 18:1 $\Delta^{11}$, vaccenic acid; ND, not detected.

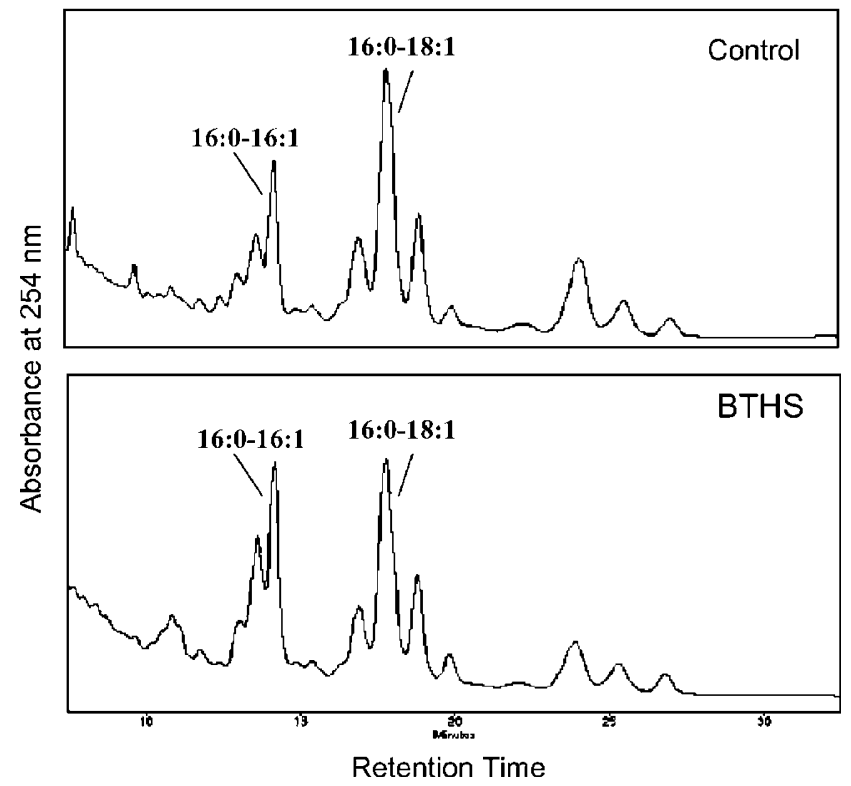

Figure 1 High-performance liquid chromatography of diacylglycerol species derived from lymphoblast PC. PC was isolated from lymphoblasts of BTHS patients and controls. PC was converted to 1,2-diacyl-3-(3',5'-dinitrobenzoyl)-glycerol and analyzed by reversed-phase high-performance liquid chromatography. ${ }^{20} 16: 0$ 16:1, palmitoyl-palmitoleoyl-PC; 16:0-18:1, palmitoyl-oleoyl-PC.

In general, there was no appreciable difference between the fatty acid compositions of mitochondrial and microsomal PC (Table 2).

The fatty acid composition of PE was almost normal in BTHS, except for a small increase in palmitoleic acid (Table 1). Again, this small alteration was detected in both mitochondria and microsomes. In all lymphoblasts, mitochondrial PE contained more stearic acid than microsomal PE (Table 2).

\section{Characterization of Mitochondria}

Since CL is specifically associated with the mitochondrial compartment, we studied the function and structural organization of lymphoblast mitochondria. First, we stained lymphoblasts with MitoTracker Red CMXRos to visualize the distribution of mitochondria within the cell (Figure 2). In normal lymphoblasts, mitochondria lined the periphery of the cytoplasm and they often formed a small density at one side of the cell. This observation was consistent with previous studies, showing mitochondria in two cellular compartments, namely the narrow cytoplasmic layer surrounding the large nucleus and the space near the nuclear invagination. ${ }^{23}$

In BTHS, the polarity of the mitochondrial organization increased. Typically, there was a substantial accumulation of MitoTracker dye at one side of the cell. Flow cytometry demonstrated an overall increase of dye concentration in BTHS lymphoblasts (Figure 2). In theory, the increase in dye concentration could be the result of higher dye affinity or it could be the result of increased mitochondrial mass in BTHS. Examination of lymphoblasts by transmission electron microscopy supported the latter explanation, as it showed an impressive accumulation of mitochondria, especially near the nuclear invagination (Figure 3). Morphometric analysis confirmed a higher surface density of mitochondria and a higher number of mitochondrial cross-sections in lymphoblasts from BTHS patients (Table 3). 
Table 2 Fatty acid composition of mitochondrial and microsomal phospholipids from lymphoblasts

\begin{tabular}{|c|c|c|c|c|c|c|c|c|}
\hline \multirow[t]{3}{*}{ Fatty acid } & \multicolumn{4}{|c|}{$P C$} & \multicolumn{4}{|c|}{$P E$} \\
\hline & \multicolumn{2}{|c|}{ Mitochondria } & \multicolumn{2}{|c|}{ Microsomes } & \multicolumn{2}{|c|}{ Mitochondria } & \multicolumn{2}{|c|}{ Microsomes } \\
\hline & Control & BTHS & Control & BTHS & Control & BTHS & Control & BTHS \\
\hline $14: 0$ & $2.5 \pm 0.3$ & $3.6 \pm 0.1$ & $2.0 \pm 0.2$ & $2.8 \pm 0.4$ & $2.0 \pm 0.7$ & $3.1 \pm 0.1$ & $5.8 \pm 0.1^{\mathrm{b}}$ & $6.7 \pm 1.7$ \\
\hline $16: 0$ & $27.2 \pm 1.6$ & $28.0 \pm 1.9$ & $26.6 \pm 1.9$ & $25.7 \pm 3.6$ & $6.4 \pm 0.5$ & $5.6 \pm 0.1$ & $3.6 \pm 0.4^{\mathrm{b}}$ & $3.8 \pm 1.2$ \\
\hline $16: 1$ & $6.8 \pm 0.1$ & $10.1 \pm 1.1^{\mathrm{a}}$ & $6.1 \pm 0.6$ & $10.4 \pm 0.8^{\mathrm{a}}$ & $2.6 \pm 0.4$ & $3.7 \pm 0.1^{\mathrm{a}}$ & $2.1 \pm 0.2$ & $3.7 \pm 0.2^{\mathrm{a}}$ \\
\hline 18:0 & $14.4 \pm 0.4$ & $12.6 \pm 1.1$ & $14.1 \pm 2.7$ & $13.9 \pm 3.2$ & $26.3 \pm 0.2$ & $25.3 \pm 0.5$ & $15.7 \pm 0.5^{\mathrm{b}}$ & $14.4 \pm 0.3^{\mathrm{b}}$ \\
\hline $18: 1 \Delta^{9}$ & $29.1 \pm 1.2$ & $24.6 \pm 1.5^{\mathrm{a}}$ & $28.8 \pm 0.6$ & $26.7 \pm 1.4$ & $24.7 \pm 3.2$ & $25.2 \pm 0.4$ & $25.3 \pm 3.0$ & $29.5 \pm 0.1$ \\
\hline $18: 1 \Delta^{11}$ & $6.2+0.5$ & $8.4+1.6$ & $6.7+0.1$ & $7.1+2.0$ & ND & ND & ND & ND \\
\hline $18: 2$ & $1.7 \pm 0.1$ & $1.8 \pm 0.3$ & $1.8 \pm 0.1$ & $1.7 \pm 0.2$ & $1.2 \pm 0.2$ & $1.6 \pm 0.1$ & $1.5 \pm 0.2$ & $1.6 \pm 0.1$ \\
\hline $20: 1$ & $2.2 \pm 0.4$ & $1.6 \pm 0.2$ & $2.4 \pm 0.1$ & $1.8 \pm 0.1$ & $1.7 \pm 0.3$ & $1.4 \pm 0.1$ & $1.6 \pm 0.1$ & $1.6 \pm 0.1$ \\
\hline $20: 2$ & $1.7 \pm 0.1$ & $1.8 \pm 0.3$ & $2.0 \pm 0.1$ & $1.8 \pm 0.1$ & $1.3 \pm 0.1$ & $1.8 \pm 0.1$ & $1.6 \pm 0.1$ & $2.0 \pm 0.1$ \\
\hline $20: 3$ & $2.8 \pm 0.3$ & $3.1 \pm 0.1$ & $3.2 \pm 0.1$ & $3.4 \pm 0.3$ & $6.2 \pm 0.9$ & $8.4 \pm 0.4$ & $8.6 \pm 0.9$ & $8.5 \pm 0.2$ \\
\hline $20: 4$ & $2.7+0.2$ & $2.4+0.6$ & $2.8+0.2$ & $2.5+0.6$ & $12.5+0.9$ & $11.4+0.7$ & $14.4+0.3$ & $13.5+1.1$ \\
\hline $22: 5$ & $0.7 \pm 0.2$ & $0.6 \pm 0.1$ & $0.8 \pm 0.2$ & $0.6 \pm 0.1$ & $3.8 \pm 1.0$ & $3.2 \pm 0.4$ & $5.4 \pm 1.8$ & $3.4 \pm 1.0$ \\
\hline $22: 6$ & $0.8 \pm 0.1$ & $0.7 \pm 0.1$ & $0.8 \pm 0.2$ & $0.6 \pm 0.1$ & $6.3 \pm 0.8$ & $5.6 \pm 0.1$ & $7.0 \pm 1.9$ & $5.2 \pm 0.9$ \\
\hline Others & $1.0 \pm 0.1$ & $0.8 \pm 0.2$ & $1.7 \pm 0.8$ & $1.1 \pm 0.4$ & $5.0 \pm 0.1$ & $3.7 \pm 0.1$ & $7.5 \pm 0.4$ & $6.0 \pm 1.1$ \\
\hline
\end{tabular}

Fatty acid composition is given in mass $\%$. Fatty acids are abbreviated as $x: y$, where $x$ represents the number of carbon atoms and $y$ represents the number of double bonds.

${ }^{\mathrm{a}}$ Significant difference between control and BTHS $(P<0.05)$.

${ }^{\mathrm{b}}$ Significant difference between mitochondria and microsomes $(P<0.05)$.

18:1 $\Delta^{9}$, oleic acid; $18: 1 \Delta^{11}$, vaccenic acid; ND, not detected.

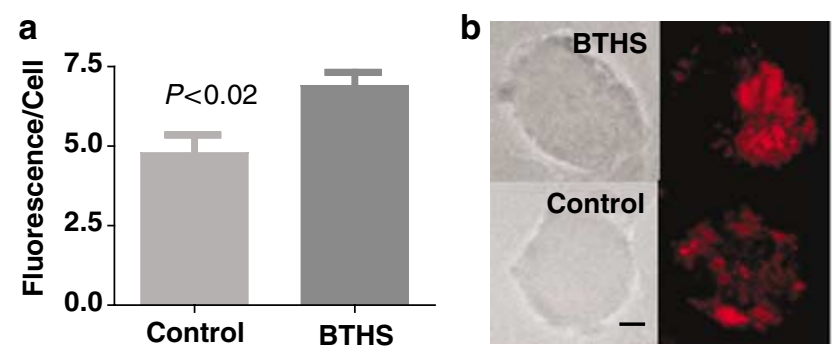

Figure 2 Staining of lymphoblast mitochondria with MitoTracker RedCMXRos. Lymphoblasts were stained with MitoTracker RedCMXRos and analyzed by flow cytometry (a) and confocal fluorescence microscopy (b). Fluorescence yield is given in arbitrary units. Microscopic images include native light images (left panel) and confocal 3D fluorescence images reconstructed from multiple $0.2 \mu \mathrm{m}$ sections (right panel). $\mathrm{Bar}=3 \mu \mathrm{m}$.

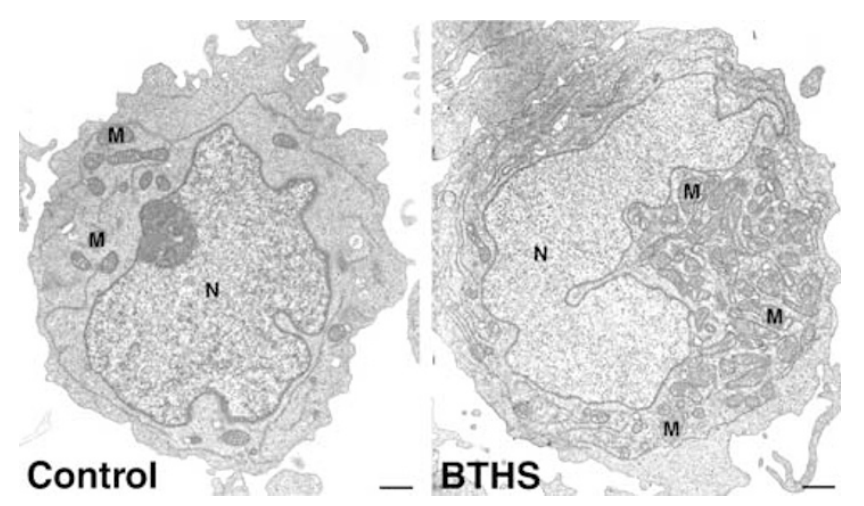

Figure 3 Electron micrographs of lymphoblasts from BTHS patients and controls. BTHS lymphoblasts show hyperproliferation of mitochondria. $\mathrm{Bar}=1 \mu \mathrm{m}$. $\mathrm{M}$, mitochondria; $\mathrm{N}$, nucleus.
Next, we determined the ability of lymphoblasts to perform oxidative phosphorylation. Mitochondrial membrane potential $\left(\Delta \Psi_{\mathrm{m}}\right)$ was monitored with the fluorescence probe JC- 1 . We found a reduction of $\Delta \Psi_{\mathrm{m}}$ by about $30 \%$ in BTHS lymphoblasts compared to controls (Figure 4). Furthermore, we measured the rate of mitochondrial ATP formation. For this purpose, lymphoblasts were permeabilized with digitonin and incubated in an assay buffer that contained inhibitors of other ATP synthetic or consuming reactions, including glycolysis, adenylate kinase, and ATP hydrolysis. ${ }^{21}$ We found that the rate of mitochondrial ATP formation was similar in lymphoblasts from BTHS patients and controls (Figure 5).

\section{Discussion}

In this study, we used lymphoblasts to characterize mitochondrial properties of patients with BTHS. We found hyperproliferation of mitochondria combined with abnormalities in energy metabolism, supporting the idea that BTHS is a mitochondrial disease. ${ }^{24}$ Dysfunction of mitochondria provides a plausible explanation for some of the most common symptoms of BTHS, such as dilated cardiomyopathy and skeletal muscle weakness.

Involvement of mitochondria in BTHS has been suspected ever since the disease was described, mainly because of reduced cytochrome concentrations and diminished respiratory enzyme activities in skeletal muscle biopsies from affected individuals. ${ }^{1,25-27}$ However, it has been difficult to 
Table 3 Morphometric analysis of lymphoblast mitochondria by transmission electron microscopy

\begin{tabular}{llcc}
\hline Variable & Unit & Control & BTHS \\
\hline Mitochondrial cross-sections & Number per cell & $11 \pm 2$ & $24 \pm 6^{*}$ \\
Mitochondrial surface density & Percent of whole cell & $5.0 \pm 0.5$ & $9.7 \pm 1.0^{*}$ \\
& Percent of cytoplasm & $7.9 \pm 0.7$ & $14.4 \pm 1.1^{*}$ \\
\hline
\end{tabular}

Asterisks indicate a significant difference between control and BTHS $(P<0.05)$.

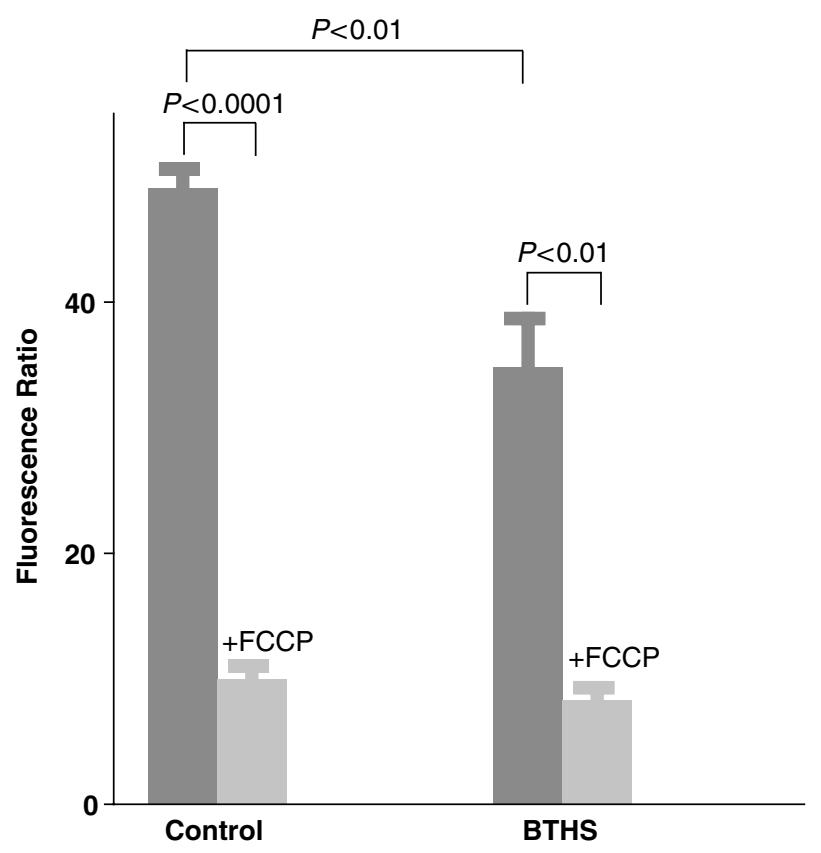

Figure 4 Mitochondrial membrane potential $\left(\Delta \Psi_{\mathrm{m}}\right)$ in lymphoblasts. $\Delta \Psi_{\mathrm{m}}$ was monitored by flow cytometry after staining of lymphoblasts with the dye JC- 1 . The ratio of red fluorescence (JC-1 aggregates) over green fluorescence (JC-1 monomers) was calculated as a surrogate of $\Delta \Psi_{\mathrm{m}}$. Carbonyl cyanide $p$-(trifluoromethoxy)phenylhydrazone (FCCP) was added to determine the fluorescence ratio of uncoupled mitochondria $\left(\Delta \Psi_{\mathrm{m}}=0\right)$.

demonstrate the specificity of these changes in relation to BTHS. Thus, definite conclusions about the mitochondrial pathology could not be drawn, although it appeared that the mitochondrial dysfunction was not caused by a defect in any specific respiratory enzyme. ${ }^{3}$ This concept was consistent with the lipid etiology of BTHS. ${ }^{6,7,9}$ Since phospholipids form the general environment of membrane proteins, they may influence the performance of multiple respiratory enzymes. The phospholipid most affected by the disease was CL, which underwent a complete reorganization of its fatty acid pattern. Specific involvement of CL corresponded with the mitochondrial localization of tafazzin. ${ }^{28}$

However, the lipid abnormalities in BTHS were not confined to CL. PC showed a distinct alteration of its fatty acid composition, which, although less drastic than in CL, was consistently present in lymphoblasts from BTHS patients. The compositional change in lymphoblast PC, namely a shift from 16:0-18:1-PC to 16:0-16:1-PC, supports our

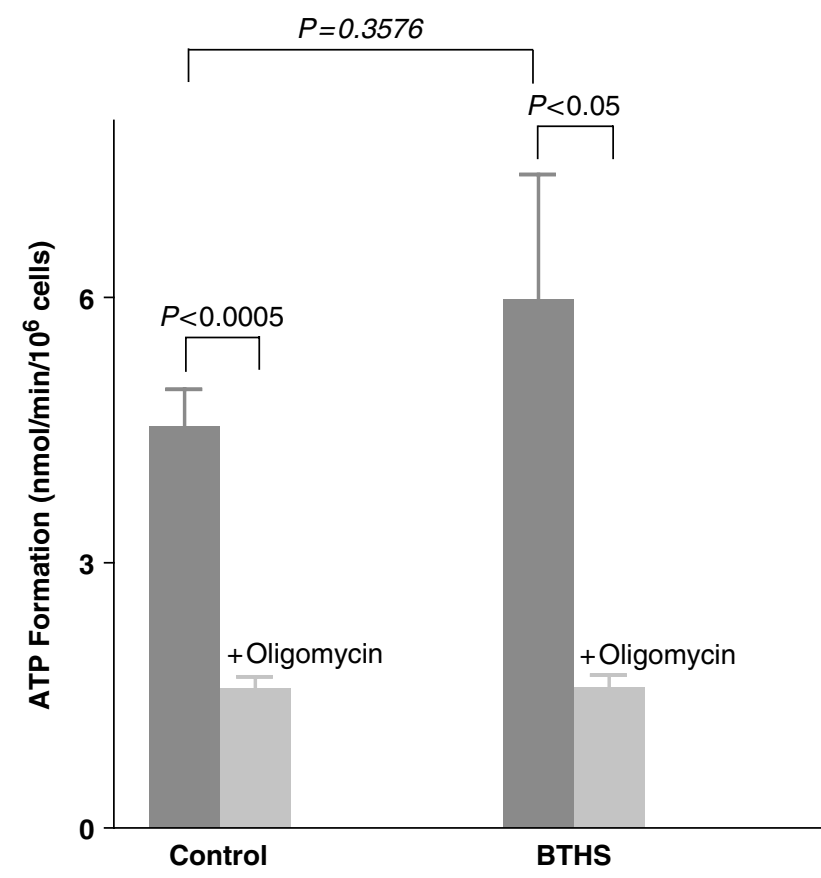

Figure 5 Rate of ATP formation in permeabilized lymphoblasts. Cells were permeabilized with digitonin and the rate of ATP formation was measured in the absence and presence of oligomycin.

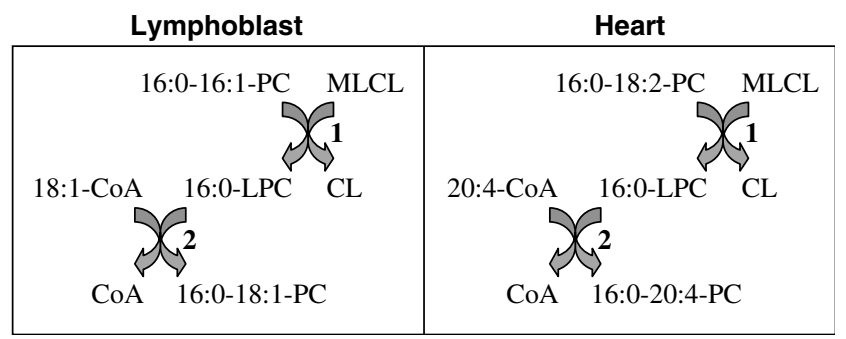

Figure 6 Mitochondrial acyl transfer reactions. The scheme shows a sequence of acyl transfer reactions in which PC donates a fatty acid to CL (step 1) and is subsequently reacylated with another fatty acid (step 2). The proposed reactions explain the changes in PC composition observed in patients with BTHS. In lymphoblasts, BTHS caused increase of 16:0-16:1-PC and decrease of 16:0-18:1-PC due to inhibition of the step 1 reaction. Likewise, BTHS caused increase of 16:0-18:2-PC and decrease of 16:0-20:4-PC in heart. $^{10}$ LPC, lysophosphatidylcholine; MLCL, monolysocardiolipin.

hypothesis that acyl groups are transferred from PC to CL for the purpose of fatty acid remodeling. ${ }^{15}$ As shown in Figure 6, we are proposing a reaction 
sequence, in which $\mathrm{PC}$ donates 16:1 residues to monolyso-CL, followed by reacylation of lyso-PC with 18:1. In BTHS, where the PC/CL transacylation is inhibited, our model predicts a rise of 16:1 in PC, a decline of 16:1 in CL, and a decline of 18:1 in PC. This is exactly what we found in BTHS lymphoblasts. The model is also applicable to heart, where we described complementary changes in PC and CL of two BTHS patients. ${ }^{10}$ In these two patients, cardiac 16:0-18:2-PC increased at the expense of 16:0-20:4-PC. This, together with the lack of 18:2 in CL, suggested that 18:2 was transferred from PC to CL and 20:4 was used to reacylate lyso-PC (Figure 6).

Although our data supported the notion that the metabolic defect of BTHS was localized in mitochondria, the same compositional change of PC was found in microsomes. It is possible that tafazzin is also involved in phospholipid remodeling outside of mitochondria, but a more likely interpretation of the microsomal data is that mitochondrial and microsomal PCs are in equilibrium. Rapid exchange of lipids between membranes may be mediated by lipid transfer proteins ${ }^{29}$ or it may occur at contact sites between mitochondria and the endoplasmic reticulum. ${ }^{30}$ Finally, crosscontamination during the isolation of mitochondrial and microsomal fractions may contribute to an equilibration of the molecular species.

The lipid abnormalities led to a compromise of bioenergetic coupling in lymphoblast mitochondria from BTHS patients. Impaired coupling was also observed in tafazzin-deficient yeast, where Ma et $a l^{28}$ found a decrease of the $\mathrm{P} / \mathrm{O}$ ratio and the respiratory control ratio. Their data suggested low coupling efficiency, presumably due to the inability to maintain $\Delta \Psi_{\mathrm{m}}$. Our data directly confirmed that $\Delta \Psi_{\mathrm{m}}$ was low in BTHS patients.

However, oxidative ATP formation of BTHS lymphoblasts was normal, a finding that may be explained by the increase in mitochondrial mass. Since ATP formation was measured in digitonintreated cells, it is also possible that the experimental protocol altered the properties of the inner mitochondrial membrane to an extent that would obscure any difference between BTHS and controls. Nevertheless, mitochondrial proliferation, mainly near the nuclear invagination, is consistent with the observation of preserved ATP production in the face of low $\Delta \Psi_{\mathrm{m}}$. Abnormal proliferation of mitochondria is a pathologic hallmark of mitochondrial myopathies. ${ }^{24}$ This hyperproliferation is generally thought to compensate for deficiencies in mitochondrial energy production. Its presence in BTHS reaffirms that the disease belongs to the broad category of mitochondrial disorders.

\section{Acknowledgement}

This work was supported in part by a grant-in-aid from the American Heart Association.

\section{References}

1 Barth PG, Scholte HR, Berden JA, et al. An X-linked mitochondrial disease affecting cardiac muscle, skeletal muscle and neutrophil leucocytes. J Neurol Sci 1983;62:327-355.

2 Kelley RI, Cheatham JP, Clark BJ, et al. X-linked dilated cardiomyopathy with neutropenia, growth retardation, and 3-methylglutaconic aciduria. J Pediatr 1991;119: 738-747.

3 Barth PG, Wanders RJA, Vreken P, et al. X-linked cardioskeletal myopathy and neutropenia (Barth syndrome) (MIM 302060). J Inherit Metab Dis 1999;22: 555-567.

4 Barth PG, Valianpour F, Bowen VM, et al. X-linked cardioskeletal myopathy and neutropenia (Barth syndrome): an update. Am J Med Genet 2004;126A: 349-354.

5 Bione S, D'Adamo P, Maestrini E, et al. A novel Xlinked gene, G4.5. is responsible for Barth syndrome. Nat Genet 1996;12:385-389.

6 Neuwald AF. Barth syndrome may be due to an acyltransferase deficiency. Curr Biol 1997;7:R465-R466.

7 Vreken P, Valianpour F, Nijtmans LG, et al. Defective remodeling of cardiolipin and phosphatidylglycerol in Barth syndrome. Biochem Biophys Res Comm 2000; 279:378-382.

8 Schlame M, Rua D, Greenberg ML. The biosynthesis and functional role of cardiolipin. Progr Lipid Res 2000;39:257-288.

9 Schlame M, Towbin JA, Heerdt PM, et al. Deficiency of tetralinoleoyl-cardiolipin in Barth syndrome. Ann Neurol 2002;51:634-637.

10 Schlame M, Kelley RI, Feigenbaum A, et al. Phospholipid abnormalities in children with Barth syndrome. J Am Coll Cardiol 2003;42:1994-1999.

11 Valianpour F, Wanders RJA, Barth PG, et al. Quantitative and compositional study of cardiolipin in platelets by electrospray ionization mass spectrometry. Clin Chem 2002;48:1390-1397.

12 Kuijpers TW, Maianski NA, Tool ATJ, et al. Neutrophils in Barth syndrome (BTHS) avidly bind annexin$\mathrm{V}$ in the absence of apoptosis. Blood 2004;103: 3915-3923.

13 Vaz FM, Houtkooper RH, Valianpour F, et al. Only one splice variant of the human TAZ gene encodes a functional protein with a role in cardiolipin metabolism. J Biol Chem 2003;278:43089-43094.

$14 \mathrm{Gu}$ Z, Valianpour F, Chen S, et al. Aberrant cardiolipin metabolism in the yeast taz1 mutant: a model for Barth syndrome. Mol Microbiol 2004;51:149-158.

$15 \mathrm{Xu} \mathrm{Y,} \mathrm{Kelley} \mathrm{RI,} \mathrm{Blanck,} \mathrm{TJJ,} \mathrm{et} \mathrm{al.} \mathrm{Remodeling} \mathrm{of}$ cardiolipin by phospholipid transacylation. J Biol Chem 2003;278:51380-51385.

16 Towbin JA, Bowles NE. The failing heart. Nature 2002;415:227-233.

17 Schoenberger J, Seidman CE. Many roads lead to a broken heart: the genetics of dilated cardiomyopathy. Am J Hum Genet 2001;69:249-260.

18 Trounce IA, Kim YL, Jun AS, et al. Assessment of mitochondrial oxidative phosphorylation in patient muscle biopsies, lymphoblasts, and transmitochondrial cell lines. Methods Enzymol 1996;264: 484-508.

19 Bligh EG, Dyer WJ. A rapid method of total lipid extraction and purification. Can J Biochem Physiol 1959;37:911-917. 
20 Takamura H, Narita H, Urade R, et al. Quantitative analysis of polyenoic phospholipid molecular species by high performance liquid chromatography. Lipids 1986;21:356-361.

21 Ouhabi R, Boue-Grabot M, Mazat J P. Mitochondrial ATP synthesis in permeabilized cells: assessment of ATP/O values in situ. Anal Biochem 1998;263:169-175.

22 Williamson JR, Corkey BE. Assay of intermediates of the citric acid cycle and related compounds by fluorometric enzyme methods. Methods Enzymol 1969;13:434-513.

23 Manteifel V, Bakeeva L, Karu T. Ultrastructural changes in chondriome of human lymphocytes after irradiation with He-Ne laser: appearance of giant mitochondria. J Photochem Photobiol B 1997;38:25-30.

24 DiMauro S. Mitochondrial diseases. Biochim Biophys Acta 2004;1658:80-88.

25 Figarella-Branger E, Pellissier JF, Scheiner C, et al. Defects of the mitochondrial respiratory chain complexes in three pediatric cases with hypotonia and cardiac involvement. J Neurol Sci 1992;108: 105-113.

26 Ades LC, Gedeon AK, Wilson MJ, et al. Barth syndrome: clinical features and confirmation of gene localization to distal Xq28. Am J Med Genet 1993; 45:327-334.

27 Christodoulou J, McInnes RR, Jay V, et al. Barth syndrome: clinical observation and genetic linkage studies. Am J Med Genet 1994;50:255-264.

28 Ma L, Vaz FM, Gu Z, et al. The human TAZ gene complements mitochondrial dysfunction in the yeast taz1 $\Delta$ mutant. J Biol Chem 2004;279:44394-44399.

29 Wirtz KWA. Phospholipid transfer proteins revisited. Biochem J 1997;324:353-360.

30 Rizzuto R, Pinton P, Carrington W, et al. Close contacts with the endoplasmic reticulum as determinants of mitochondrial $\mathrm{Ca}^{2+}$ responses. Science 1998; 280:1763-1766. 